\title{
Design and Realization of PLC Control System for Magnetic Iron Ore Separating Equipment
}

\author{
Wei Ren \\ Department of Automation, Tangshan Industrial Vocational Technical College, Hebei, 063000, \\ China
}

Keywords: Electromagnetic separator. PID self-optimizing control. I/O interface. Software design.

\begin{abstract}
Magnetic iron concentrate beneficiation process, in order to improve the grade of iron concentrate powder, electromagnetic magnetic separation equipment can be used to realize. The use of PLC technology, PID control of self-optimization control algorithm, by controlling the magnetic field intensity and concentration of iron ore powder in the magnetic separation barrel, the grade of iron concentrate can be improved, to meet the requirements of iron concentrate grade in production process. The production process and parameters displayed and controlled by the HMI man-machine interface. After the system is put into use, the new equipment was compared with the permanent magnetic separation equipment, the data show, performance of this equipment is better than conventional permanent magnetic separator.
\end{abstract}

\section{Introduction}

In magnetic iron ore powder cleaning process, use of PLC, iron concentration detection sensor, according to the concentration of ore pulp inflow and outflow, using the PID self-optimizing control algorithm, adjust automatically the regulating valves of water supply or drainage and control intensity of magnetic fields, to make the electromagnetic separator run in the best condition. So that the outflow of the iron pulp grade can be improved, to meet the requirements of iron concentrate grade in production process. At the same time the process display and control is realized by using the HMI touch screen man-machine interface.

\section{Iron concentrate beneficiation process}

Magnetic iron ore sorting equipment structure see Fig. 1 (1) buffer tank (2) overflow tank (3) Magnetic barrel (4) Junction box (5) Mounting flange (6) outer cylinder (7) water supply ring (8) concentration sensor). The concentration of $60 \%$ ore pulp by selecting machine through the buffer tank into the upper end of magnetic separation barrel, and the upward flow of wash water flow reversely. Wash water from the water supply ring at the lower end of the Magnetic barrel inflow along the tangential direction. The water supply pressure is $0.2-0.3 \mathrm{Mpa}$. Water with $2 \sim 6 \mathrm{~cm} / \mathrm{s}$ speed up in the separation barrel. The water wash the falling ore pulp. Due to the proportion is less gangue than iron, gangue rise to the overflow tank with water discharge in the action of upward flowing water. In order to avoid being the rising small particles of iron washed away by water, the iron concentration in the overflow ore exceed the standard, thus making the use of electromagnetic coil generates magnetic fields, so that the iron powder have gathered under the action of magnetic field, magnetic agglomeration of iron powder fall down by gravity, flow out from the bottom outlet. The finished product is discharged the grade reached more than 64\%. 


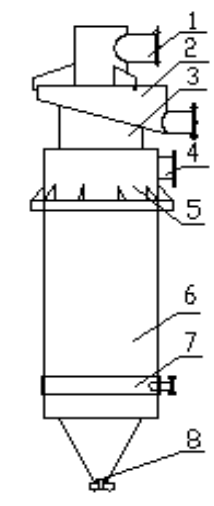

Fig. 1: Structure of magnetic ore separator

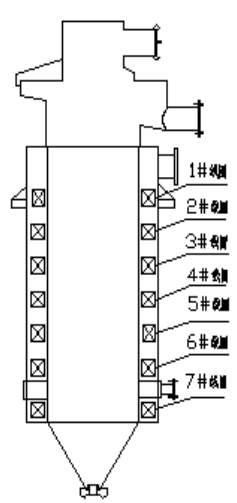

Fig. 2: Distribution of electromagnetic coils

\section{The hardware design of the control system}

\section{Separating barrel magnetic fields design}

According to the general principle of electric coil generates a magnetic field strength of the magnetic field, can produce controllable magnetic field. Based on the characteristics of the iron separation, magnetic separation barrel is divided into constant magnetic field and alternating magnetic field region, 1 s shown as figure 2. Set the $1 \# \sim 7 \#$ of seven groups of electromagnetic coils in the magnetic outer side of the barrel from top to bottom [14]. Set the 1\# electromagnetic coil in a constant magnetic field area, produce the Constant magnetic field lines; others six layer disposed electromagnetic coils in the top-down alternating magnetic field area, with a jump of one or two parallel each other, constitute the three group separating coils. They can generate three groups of circular magnetic field.

Set a constant magnetic field coil's role: To make magnetic powder to produce magnetic agglomeration, increase the magnetic force downward, prevent iron overflow along the upper overflow tank, overflow proportional control of small particles of iron powder, iron loss reduction ratio [10].

Set three sets of alternating magnetic field coil's role: Three groups of magnetic coils turns intermittent electrified, the magnetic field of the coil produces cycle down on coil internal. By controlling the electric current, the electrifying time and electrifying time interval, control the grade of finished product ore [11].

\section{The principle of control system.}

DC power supply electromagnetic coil: In order to respectively produce controllable constant magnetic field and the sequence of moving down recycle pulsating magnetic field in a constant magnetic field region and the alternating magnetic field region. Electromagnetic coils adopts a controllable DC power supply [12].

Control the magnetic field strength of the constant region: A according to the iron concentration of overflow regulate the intensity of the magnetic field, the iron particles outflow concentration meet the production process.

Control the magnetic field strength of the alternating magnetic field region: The concentration sensor detection is arranged at the lower part of the overflow liquid level. According to the detection of iron ore concentration, the strength of the magnetic field in three sets of alternating electromagnetic coils, the electrifying period from top to bottom turns was controlled, each group of 1.7 seconds. When it is detected that the overflow liquid level lower concentration increases, then increasing the working current of the electromagnetic coil, the magnetic field intensity increases; on the other hand, decrease the work current of the electromagnetic coil.

PID self-optimizing control on ore concentration, ore discharge amount, quantity of water supply: In the production of real-time change into ore concentration, in order to ensure the ore grade and save water, must be integrated into the automatic control volume of ore output, water supply. Therefore, the concentration sensor is arranged in the export of magnetic barrel lower, set the 
regulating valves in the water entering port and a mine outlet, according to the ore concentration, discharge concentration, using the PID self-optimizing control algorithm, to achieve water supply regulating valve, ore discharge regulating integrated automatic control[9, 15].

\section{The Design of the System Hardware}

The control system consists of a S7-CPU 224XP PLC, the analog input module EM231, analog output module EM232, SMART 700 HMI human-machine interface, phase-shift voltage regulation module, rectifier module, input concentration sensor, output concentration sensor, water electric regulating valve, export electric regulating valve[1,2,13]. An multiple variable an integrative control system is shown as Figure 3.

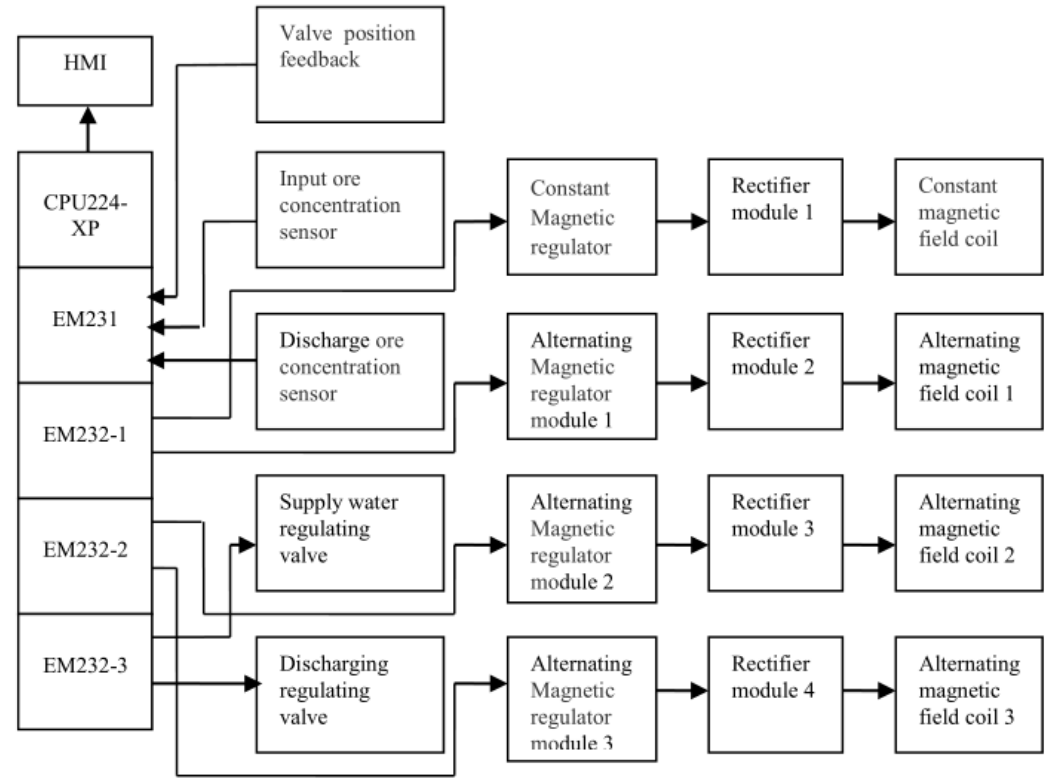

Fig. 3: Structure of the control system

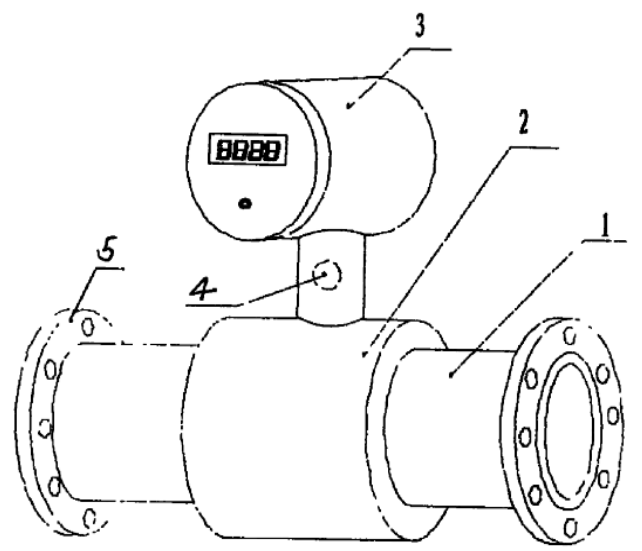

Fig. 4: Iron density detection sensor (1)Main pipeline (2)Sensor coil sheath (3)Transducer (4)Wiring terminal (5) Flange)

Selection by iron concentration detection device 1s shown as figure 4. By adopting the electromagnetic principle, according to the changes through the medium of magnetic material, accurate measurement of the amount of change in iron in ore pulp [5, 6, 7].

The analog input modules: The EM231 module has four analog input Channels, using two wire 4-20mA input signal, the circuit principle is shown as Figure 5.Two concentration sensor signals, position feedback signal of electric regulating valves, access to the EM231 input ports [3]. 


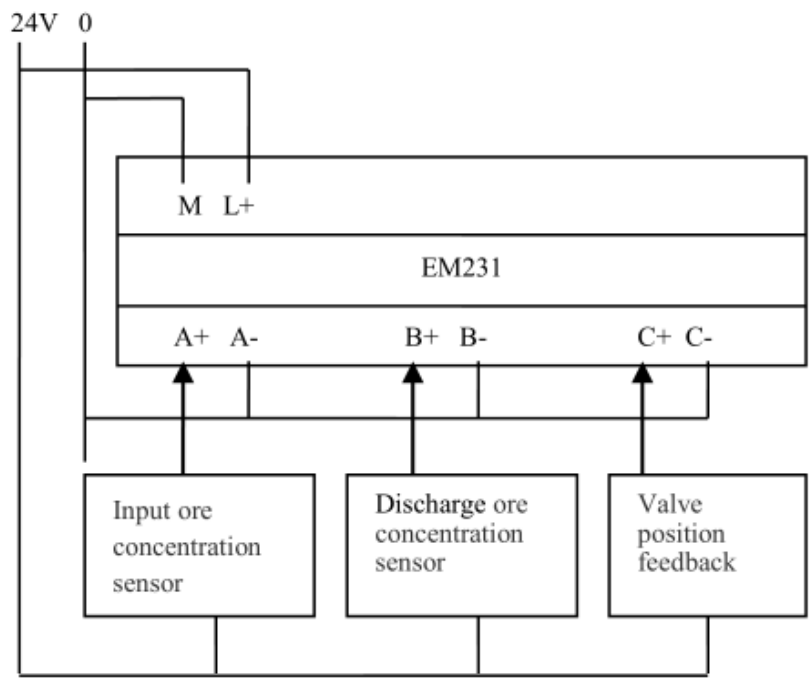

Fig. 5: Analog input signal measuring circuit

The analog output modules: Three analog output modules EM232 Selected, output signal 020mA. EM231-1, EM231-2, control voltage regulator modules and the strength of the magnetic field separation barrel two areas. The principle is show as figure 6 .

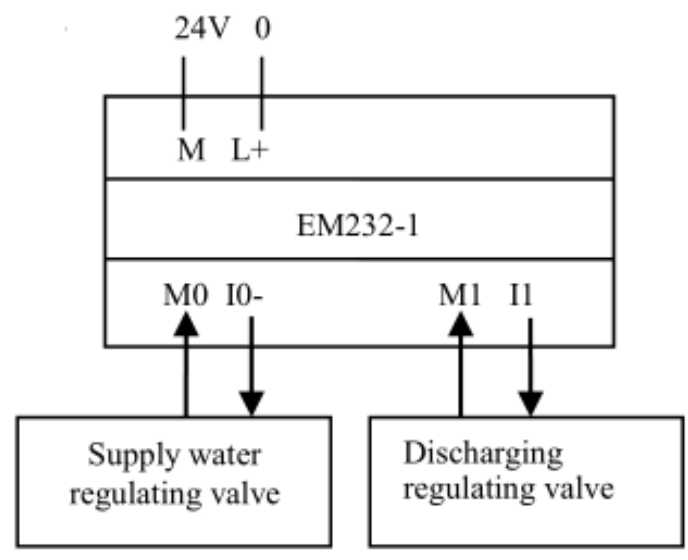

Fig. 6: The control principle of magnetic field

EM231-3 control the opening degree of the water supply regulating valve and the flow out of the mine electric regulating valve. The principle show in figure 7.

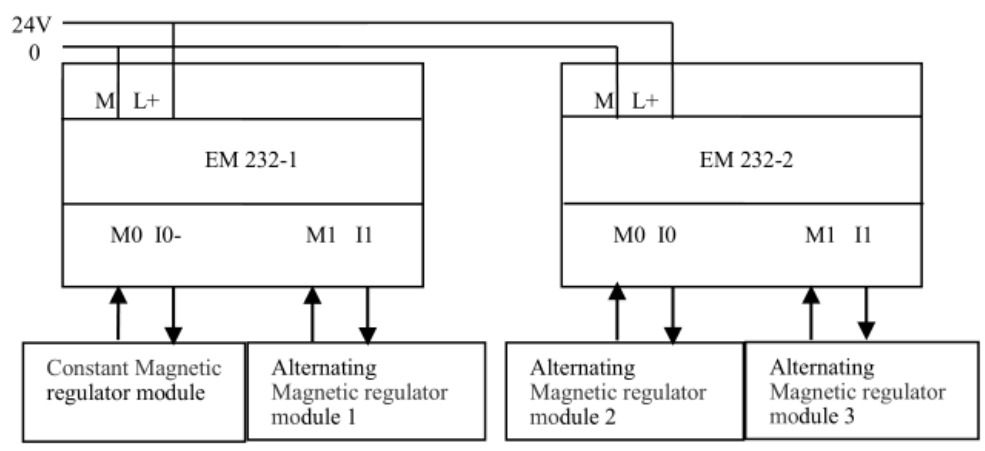

Fig. 7: The control principle of electric regulating valve

\section{The design of PLC control system software}

Control flow chart of PLC control system is shown as Figure 8[9, 15]. 


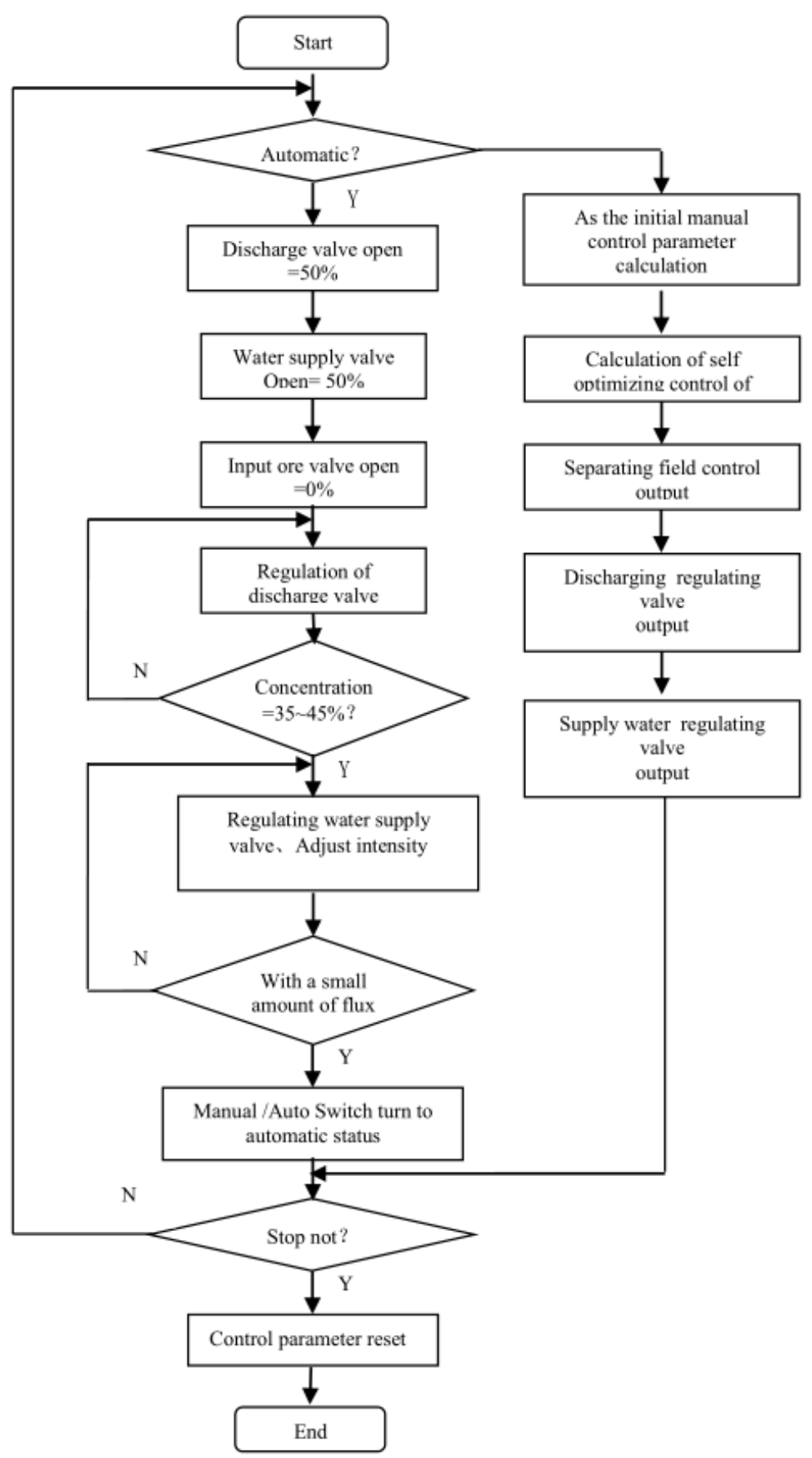

Fig. 8: The PLC control software flow

\section{PLC control system using effect}

The PLC automatic control system is used in a mineral processing plant in the production. This electromagnetic separator with permanent magnetic separator are compared, the data is shown as Table 1.

Table 1: Comparison of usage data

\begin{tabular}{|c|c|c|c|c|}
\hline Grade(\%) & Input ore pulp & $\begin{array}{c}\text { Overflow } \\
\text { quality }\end{array}$ & The concentrate & $\begin{array}{c}\text { The percentage of } \\
\text { grade }\end{array}$ \\
\hline $\begin{array}{c}\text { PLC automatic } \\
\text { electromagnetic } \\
\text { separator }\end{array}$ & 60.53 & 46.89 & 65.20 & 4.67 \\
\hline $\begin{array}{c}\text { Permanent magnetic } \\
\text { separator }\end{array}$ & 60.53 & 52.25 & 63.00 & 2.47 \\
\hline
\end{tabular}


Analysis of the data in Table 1 draws the conclusion as follows: The concentrate grade of the electromagnetic machine is $65.2 \%$, the permanent magnetic separator is $63 \%$, the former increased by $2.2 \%$ than the latter; The concentrate grade increased percentage is $4.67 \%$ for the electromagnetic machine, the grade increased percentage is $2.47 \%$ for permanent magnetic separator, the former increased by $2.2 \%$ than the latter. Comparison of the data shows that, the performance of automatic electromagnetic separator is better than that of conventional permanent magnetic separator.

\section{References}

[1] Liu Hua-Bo, Siemens S7-200 PLC Programming and application of case selection. Beijing: China Machine Press, 2009.

[2] Xiao Bao-Xing, Siemens S7-200 PLC The use of experience and skills, Beijing: China Machine Press, 2011.

[3] Zhang Yang and Cai Chun-Wei, S7-200 PLC The principle and application system design, Beijing: China Machine Press, 2007.

[4] Qiu Li-Cun and Wu Yong-Zhong, Development of inductive magnetic medium meter, Journal of Hebei Institute of Technology, 25, pp.35-38, 2003.

[5] Qiu Li-Cun, Using magnetic measuring content of sinusoidal excitation, Industry and Mine Automation,4, pp.32-35, 2005.

[6] Zhang Shu-Wen, Research on the practice of CG type magnetic content meter, Coal Preparation Technology, 3, pp.34-37, 1994.

[7] Wang Lei, Research on weighing type slurry concentration online detection device, Journal of Kunming Science of Technology, 2008.

[8] Ma Yan and Wang Jian-Min, Application of radio frequency admittance technique in the detection of content of magnetic substance, Industry and Mine Automation, 9, pp.28-32, 2009.

[9] Wang Qiang, Automatic controller of magnetic separation column, Non-ferrous Mining and Metallurgy, 21(4), pp.51-53, 2005.

[10] Li Ming-Yong, Cheng Min, Analysis and calculation of winding losses of high frequency inductors, Power Electronics, 5, pp.38-42, 2007.

[11] Yang Chao, Magnetic separator technology progress and development direction of the weak in China, Gansu Metallurgy, 2, pp.32-35, 2010.

[12] Yuan Zhi-Tao, Development and experiment of pulse vibrating magnetic field magnetic column, Metal Mine,3, pp.36-38, 2001.

[13]Xiang Xiao-Han, Siemens PLC advanced application examples, Beijing:China Machine Press,7, pp.14-40,2010.

[14]Wang Chang-Ren, Electromagnetic Separation of Ore, Beijing:Metallurgical Industry Press,5, pp.32-41,1986.

[15]Ma Xiang-xing, Design and Simulation of Fuzzy PID Controller of Self-optimization and Selfadjustment, Computer Simulation, 28(4),pp. 216-220,2011. 